EGU21-14686

https://doi.org/10.5194/egusphere-egu21-14686

EGU General Assembly 2021

(c) Author(s) 2022. This work is distributed under

the Creative Commons Attribution 4.0 License.

\title{
Encounter of Parker Solar Probe and a Comet-like Object During Their Perihelia: Simulations and Measurements
}

\author{
Jiansen He ${ }^{1}$, Bo Cui ${ }^{1}$, Liping Yang ${ }^{2}$, Chuanpeng Hou ${ }^{1}$, Lei Zhang ${ }^{1}$, Wing-Huen $\mathrm{Ip}^{3}$, Yingdong Jia ${ }^{4}$, \\ Chuanfei Dong ${ }^{5}$, Die Duan ${ }^{1}$, Qiugang Zong ${ }^{1}$, Stuart Bale ${ }^{6,7,8}$, Marc Pulupa $^{6}$, John Bonnell, Thierry \\ Dudok de Wit ${ }^{10}$, Keith Goetz ${ }^{11}$, Peter Harvey ${ }^{6}$, Robert MacDowall ${ }^{12}$, and David Malaspina ${ }^{13}$ \\ ${ }^{1}$ School of Earth and Space Sciences, Peking University, Beijing, 100871, P. R. China \\ ${ }^{2}$ State Key Laboratory of Space Weather, National Space Science Center, Chinese Academy of Sciences, Beijing, 100190, P. \\ R. China \\ ${ }^{3}$ Institute of Astronomy, National Central University, Taoyuan, 32001, Taiwan, R.O. China \\ ${ }^{4}$ IGPP and EPSS, University of California, Los Angeles, CA 90095, USA \\ ${ }^{5}$ Department of Astrophysical Sciences, Princeton University, Princeton, NJ 08544, USA \\ ${ }^{6}$ Space Sciences Laboratory, University of California, Berkeley, CA 94720-7450, USA \\ ${ }^{7}$ Physics Department, University of California, Berkeley, CA 94720-7300, USA \\ ${ }^{8}$ The Blackett Laboratory, Imperial College London, London, SW7 2AZ, UK \\ ${ }^{10}$ LPC2E, CNRS and University of Orl' eans, Orl' eans, France \\ ${ }^{11}$ School of Physics and Astronomy, University of Minnesota, Minneapolis, MN 55455, USA \\ ${ }^{12}$ Solar System Exploration Division, NASA/Goddard Space Flight Center, Greenbelt, MD, 20771 \\ ${ }^{13}$ Laboratory for Atmospheric and Space Physics, University of Colorado, Boulder, CO 80303, USA
}

Parker Solar Probe (PSP) aims at exploring the nascent solar wind close to the Sun. Meanwhile, PSP is also expected to encounter small objects like comets and asteroids. In this work, we survey the ephemerides to find a chance of recent encounter, and then model the interaction between released dusty plasmas and solar wind plasmas. On 2019 September 2, a comet-like object $322 \mathrm{P} / \mathrm{SOHO}$ just passed its perihelion flying to a heliocentric distance of $0.12 \mathrm{au}$, and swept by PSP at a relative distance as close as $0.025 \mathrm{au}$. We present the dynamics of dust particles released from $322 \mathrm{P}$, forming a curved dust tail. Along the PSP path in the simulated inner heliosphere, the states of plasma and magnetic field are sampled and illustrated, with the magnetic field sequences from simulation results being compared directly with the in-situ measurements from PSP. Through comparison, we suggest that 322P might be at a deficient activity level releasing limited dusty plasmas during its way to becoming a "rock comet". We also present images of solar wind streamers as recorded by WISPR, showing an indication of dust bombardment for the images superposed with messy trails. We observe from LASCO coronagraph that 322P was transiting from a dimming region to a relatively bright streamer during its perihelion passage, and simulate to confirm that 322P was flying from relatively faster to slower solar wind streams, modifying local plasma states of the streams. 
